\title{
El método de casos como herramienta de enseñanza. Sus resultados y reflexiones en épocas de pandemia ${ }^{1}$
}

\author{
Case method as a teaching tool. Its results and \\ reflections in pandemic times
}

\author{
Patricia Carina Calpanchay Suárez ${ }^{2}$
}

\begin{abstract}
Resumen
El proyecto «El método de casos como herramienta de enseñanza; formación y transmisión jurídico-social para docentes y estudiantes», aprobado por resolución rectoral n. ${ }^{\circ} 1362 / 18$ de la Universidad Católica de Salta (UCASAL), surgió a partir de la reflexión sobre la propia práctica docente de la Cátedra de Introducción al Derecho, comisión B3 (primer año de la carrera de Abogacía), de la Facultad de Ciencias Jurídicas. Dicha reflexión denota un compromiso del docente con la calidad de la educación; en este caso, con la educación superior. La cátedra mencionada ha venido trabajando durante varios años con casos prácticos y jurisprudencia. Este aporte tiene por propósito dar cuenta de pequeños hallazgos encontrados durante el trabajo de campo. La investigación se realizó, epistemológicamente, desde las ciencias de la educación y el derecho y, específicamente, desde la pedagogía jurídica.
\end{abstract}

Palabras clave: casos - complementariedad - contextos virtuales - docencia

\section{Derecho/ artículo científico}

Citar: Calpanchay Suárez, P. C. (2021). El método de casos como herramienta de enseñanza. Sus resultados y reflexiones en épocas de pandemia. Omnia. Derecho y sociedad, 4 (4), pp. 47-54.

\footnotetext{
${ }^{1}$ El presente artículo se encuentra enmarcado en el proyecto de investigación «El método de casos como herramienta de enseñanza; formación y transmisión jurídico-social para docentes y estudiantes», aprobado por resolución rectoral n. ${ }^{\circ} 1362 / 18$ de la Universidad Católica de Salta. El equipo de investigación estuvo conformado por el doctor Luis Renato Rabi Baldi Cabanillas (director), Ana Mohnblatt y Patricia Calpanchay Suárez.

${ }^{2}$ Abogada, egresada de la Universidad Católica de Salta (UCASAL). Profesora en Ciencias Jurídicas egresada de la Universidad Nacional de Salta. Docente e investigadora en la Facultad de Ciencias Jurídicas de la UCASAL. pcalpanchay@ ucasal.edu.ar
} 


\section{INTRODUCCIÓN}

El aprendizaje en épocas de pandemia me obligó a repensar la práctica docente. Es así que me he planteado las siguientes preguntas: ¿cómo hacer para enseñar el derecho de un modo más próximo a la realidad profesional del abogado? ¿Se puede enseñar derecho de una manera diferente a la enseñanza tradicional? ¿Este método de enseñanza y aprendizaje que empleo a diario me permite alcanzar mis propósitos docentes? ¿Qué otras facultades de derecho del país emplean la metodología por casos?

Esta investigación analiza el contexto institucional de algunas facultades de derecho del país que emplean el método de casos y se encuentra enmarcada en el año lectivo 2019. Dichas facultades pertenecen a las universidades nacionales de Tucumán (UNT), de Buenos Aires (UBA), y las universidades Austral y Católica de Salta (UCASAL). Para la ejecución del proyecto se empleó un método mixto cuanti-cualitativo de investigación. Ello en razón de que se utilizaron distintas técnicas, a saber: entrevistas en profundidad, cuestionarios, focus group (grupo focal), observación participante y no-participante sobre determinadas situaciones áulicas, así como análisis documental.

Cada institución universitaria (más precisamente, cada facultad de derecho) está marcada por una nota de particularidad que no es otra cosa que su «cultura institucional». Leticia Piotti y Susana Lupiañez, en su obra Análisis institucional y vínculos de convivencia escolar: el desafío de construir el nosotros en las instituciones educativas, nos dicen:

Cada escuela tiene un estilo de funcionamiento, una forma particular de manifestarse y resolver los problemas que la diferen- cian de otras escuelas, aunque pertenezcan al mismo nivel y atiendan a una población de alumnos similares. Esta imagen-representación son construcciones culturales, elaboradas a lo largo de la historia institucional, en la que se entrelazan los estilos de sus directivos y docentes, cómo se interpretaron las necesidades de la comunidad, etc. (Piotti y Lupiañez, 2002, p. 18).

Margarita Poggi y Graciela Frigerio, en su obra Las instituciones educativas. Cara y ceca, analizan el concepto de cultura institucional y explican que

... cada institución posee rasgos de identidad y señas particulares que le son propios; ambos constituyen y simultáneamente son aprehensibles en lo que denominamos cultura institucional. La cultura institucional es aquella cualidad relativamente estable que resulta de las políticas que afectan a esa institución y de las prácticas de los miembros de un establecimiento. Es el modo en que ambas son percibidas por estos últimos, dando un marco de referencia para la comprensión de las situaciones cotidianas, orientando e influenciando las decisiones y actividades de todos aquellos que actúan en ella (Poggi y Frigerio, 1992, p. 35).

Analizar el concepto de cultura institucional es de vital importancia, habiendo descubierto con esta investigación sobre el método de casos que cada institución tiene sus propias particularidades, sus propios planes de estudios, programas, centros de formación docente, centros de investigación, e incluso su propia concepción filosófica sobre qué es el derecho, su propia trayectoria, sus modelos de vínculos, etc. Todos estos son componentes de la cultura institucional dentro de los cuales, en cuanto 
aquí interesa, merece especial atención la metodología de enseñanza universitaria y, dentro de ella, y en lo que constituye el específico objeto de estudio de esta investigación, el método de casos.

\section{LA COMPLEMENTARIEDAD E INSTRUMENTALIDAD DEL MÉTODO DE CASOS}

El método de casos - como objeto de la investigación- puede ser analizado desde dos puntos de vista. Desde un primer punto de vista, el método de casos se presenta en las universidades como "complementario» de la enseñanza de los contenidos teóricos. Según el diccionario de la Real Academia Española, «complementario» significa «que completa o perfecciona algo». Es un método de enseñanza complementario en la formación del abogado, lo que encuentra razón de ser en nuestro sistema jurídico continental europeo y codificado. En este sentido, en la obra coordinada por Rabbi Baldi Cabanillas (2019) Lecciones de teoría del derecho. Una visión desde la jurisprudencia constitucional, Maximiliano Consolo expresa que es importante considerar que «el aprendizaje basado en casos mira la realidad, partiendo su enseñanza de ella, bien que sin desmedro de la teoría. Esto permite inferir que en este tipo de metodología la relación entre la teoría y de la práctica es de complementariedad» (2019, p. 49). De igual modo, cuando los docentes -en las entrevistas realizadas en el transcurso de esta investigación- se refieren a «complementario de lo teórico» quieren decir que, además de la teoría que se desarrolla en cada clase, utilizan los casos prácticos o casos jurisprudenciales como una estrategia más de enseñanza.

Desde un segundo punto de vista, el método de casos tiene además un carácter instrumental: esto implica que se convierte en un instrumento para transmitir determinado contenido pedagógico e incluso un instrumento para desarrollar y potenciar habilidades cognitivas. En ese sentido, el método de casos es un «medio» para un «fin». Como medio es una herramienta, y ese fin no es otro que lograr el aprendizaje en el alumno.

\section{TIPOLOGÍA DE MÉTODOS DE CASOS}

El método de casos fue el objeto de investigación. Si bien la expresión "método de casos" puede representar socialmente (entendiendo por representaciones sociales formas de conocimiento cotidiano con anclaje en la realidad) el sistema del Common Law de los Estados Unidos, no puedo dejar de precisar que este método se aplica en las universidades del país, tal vez no de la misma manera o forma. Incluso en los EE. UU. el método fue modificándose con el correr del tiempo.

El método nació en EE. UU. y se expandió con características propias a otras regiones. En cuanto a nuestro país, las facultades de derecho que practican el método tienen construido «su» método de casos. Se trataría de un método de casos «argentinizado» e incluso, más precisamente, podríamos hablar de la existencia de una «enseñanza por casos». Ello teniendo en cuenta nuestro sistema jurídico. Esto último implica analizar el objeto de estudio desde una perspectiva del mundo de la experiencia vivida por las personas. Por ello, me propuse comprender, a través de entrevistas con los docentes, qué entendían ellos por método de casos, qué entendían por «casos», y si ellos lo aplicaban.

Un autor moderno que aborda al método conceptualmente es Sergio Palacio, en su obra Pedagogía jurídica, quien expresa que «[el método] sirve eficazmente para enseñar a los alumnos a reflexionar y razonar acerca de las

Omnia. Derecho y sociedad, núm. 4, 2021: 47-54 
decisiones judiciales como problemáticas jurídicas específicas y captar la estructura de esas decisiones. En síntesis, para enseñar el razonamiento legal» (2017, p. 134).

En igual sentido, Oscar Flores -docente de la Universidad Nacional de Tucumánen la obra Un método crítico de enseñanza y aprendizaje del derecho agrega que Langdell, en EE. UU., proponía «enseñar derecho exclusivamente a través del análisis de fallos y discusión en clase», ya que el método de casos se había iniciado «como un método científico de descubrir principios de derecho»; además, también según Langdell, «los principios del derecho, como los de toda ciencia podían conocerse por inducción. A partir de la lectura de algunos casos relevantes, se inducían las líneas generales» (Landell, citado en Flores, 2019 , p. 9). Ambos autores, junto con Rabbi Baldi Cabanillas en su obra ya citada, tienen en común una vinculación con el método de casos del «mundo anglosajón, el Common Law» (el sistema jurídico donde hizo carrera el método), pues centra su análisis especialmente en la labor de los jueces y en la relevancia de los precedentes judiciales. En efecto, se vincula al método de casos con el análisis de las sentencias judiciales (jurisprudencia). De ahí que las estructuras de las obras de los profesores Flores y Rabbi Baldi compartan el mismo esquema de abordaje en el trabajo con casos. Ese esquema es el siguiente: básicamente, se analiza una sentencia judicial (resolución judicial) de un tribunal — sea corte de justicia o tribunal ordinario de justicia-; se vincula «un contenido teórico» (tema) con el contenido de una sentencia judicial, debido a que esta última contempla determinada temática relevante en razón de la enseñanza a impartir. Esta vinculación tiene como objetivos el descubrir principios, ejercitar el pensamiento crítico del alumno, entender el razo- namiento legal. Estos objetivos se alcanzan o se procuran alcanzar a través de una serie de preguntas críticas formuladas por el docente y que interpelan al alumno. Aquí, el rol de alumno consiste en «analizar».

En otro orden de ideas o representaciones sobre el método de casos, se encuentra a autores como Juan Seda, Osvaldo Pitrau, Graciela Medina, y Mario Ackerman. En sus obras se resalta un elemento fundamental del método de casos, a saber, «el problema». En este sentido, Susana Campari en Formación pedagógica en derecho sostiene que

... un caso es un relato cuidadosamente elaborado que plantea una situación problemática extraída de la vida real, que requiere análisis, investigación, toma de decisiones y resolución y que se presenta en la clase con un objetivo didáctico previamente definido. Si no hay un problema no hay caso (Campari, 2011, p. 113).

Esta afirmación es concluyente: «Si no hay un problema no hay un caso». Y, en consecuencia, sobre este problema el alumno debe poner en ejercicio ciertas habilidades cognitivas para analizar, investigar, tomar decisiones y resolver. Otro elemento que se resalta en esta última definición es que ese problema es «extraído de la vida real» y aquí, siguiendo a Osvaldo Pitrau en Casos de derecho sucesorio, el método «encuentra su fundamento en las denominadas técnicas de simulación, basadas en reproducir situaciones semejantes a la realidad, pero dentro de un aula» (2017, p. 12). El autor agrega que «(...) y el docente hará las preguntas basadas en supuestos prácticos que simulan situaciones reales, guiando al aprendiz a encontrar la solución» (2017, p. 13). Es interesante la concepción de Pitrau sobre el método de casos que él Ilama con más 
propiedad «el método de casos prácticos»; fundamentado en las técnicas de simulación, en la mayéutica socrática (en referencia a Sócrates y Platón) y en el aprendizaje afectivo. Las obras de este segundo grupo de autores comparten en común la siguiente estructura de un «caso práctico»: un título inicial que es básicamente el tema sobre el que versa el caso. Luego consta el «planteo», que es el planteamiento de la situación problemática a resolver, el «problema». A continuación, una referencia a la jurisprudencia sobre el tópico indicando año, tomo y folio (bien que no en todos los autores se repite esta metodología). Y, por último, se expresan las tareas a realizar por el alumno. Estas «tareas» no son otra cosa que preguntas sobre las cuales el alumno no tiene una solución, sino que es él a través de su razonamiento quien debe llegar a ella. A diferencia de una sentencia judicial que consta de una solución ya dada por los jueces, aquí es el alumno quien debe llegar a dicha solución colocándose en diferentes roles, ya sea como abogado de la parte actora o de la parte demandada, e incluso en el mismo rol de juez. Lo que quisiera resaltar es que estos casos prácticos implican siempre un «hacer», más precisamente un «resolver» por parte de los alumnos.

Estas concepciones sobre método de casos o enseñanza por casos son las que fluyeron a lo largo de la investigación.

\section{FUENTES DE INVESTIGACIÓN}

En otro orden y a nivel del análisis sobre documentos y obras de la temática, cabe señalar que al comienzo, cuando se elaboró el «estado del arte» para el proyecto, no encontré material actualizado. Las obras base del proyecto fueron la de Cueto Rúa (1957) El Common Law, que analiza el método de casos en la enseñanza del derecho de los EE. UU.; su fundamentación pedagógica, sus ventajas y desventajas, efectuando algunas consideraciones sobre el tema en la Argentina; $y$, en segundo término, la obra de Selma Wassermann (1994) El estudio de casos como método de enseñanza. Sin embargo, durante el transcurso de la ejecución del proyecto de investigación se fueron encontrando distintas obras significativas de profesores argentinos, algunas actuales, otras no tanto. Es así como puedo presentar el siguiente orden trípode de bibliografía encontrada. En primer lugar, se hallan libros y artículos que abordan la enseñanza del derecho en general y, en particular, de la enseñanza por método de casos en la Argentina. Tales obras son: 1) Gordillo, Agustín (1988), El método en derecho; 2) Böhmer, Martín (1999), La enseñanza del derecho y el ejercicio de la abogacía; 3) Palacio, Sergio (2017), Pedagogía jurídica. Elementos para la formación de abogados; 4) Fischman, Fernando y Seda, Juan (2011), Formación pedagógica en derecho. Procesos comunicativos y recursos didácticos; 5) Ovejero Silva, Bruno (2018), Introducción al Common Law como sistema jurídico; y 6) Toller, Fernando (2005), Orígenes históricos de la educación jurídica con el método de casos.

En segundo lugar, encontré libros que proporcionan modelos de casos en diversas ramas del derecho, que tienen un enfoque meramente práctico y didáctico. Estas obras adoptan herramientas pedagógicas útiles tanto para alumnos como para docentes de derecho. Entre estas obras mencionamos a: 1) Medina, Graciela y Pitrau, Osvaldo (2017), Casos de derecho de familia; 2) Rolleri, Gabriel y Pitrau, Osvaldo (2017), Casos de derecho sucesorio; 3) Sancinetti, Marcelo (2016), Casos de derecho penal (tomos 1, 2 y 3); 4) Ziffer, Patricia (2011), Casos de derecho procesal penal; 5) Gullco, Hernán (2017), Casos de derecho penal especial; 6) Ackerman, Mario (2014), 
Casos y materiales del derecho del trabajo y de la seguridad social; 7) Rabbi Baldi Cabanillas, Renato (2019), Lecciones de teoría del derecho (casos de Teoría del Derecho); y 8) Flores Oscar (2019), Un método critico de enseñanza y aprendizaje del derecho.

Y por último, libros destinados a jóvenes abogados sobre cómo estudiar, plantear y argumentar un caso. Aquí puedo mencionar a Carrió, Genaro (2003), Cómo estudiar y argumentar un caso y a Suárez, Carina (2012), Cómo plantear, resolver y argumentar un caso.

\section{MOTIVOS QUE SUBYACEN EN LOS DOCENTES RESPECTO DEL USO DE LA METODOLOGÍA DE ENSEÑANZA CON CASOS}

Otro aspecto no menos relevante a analizar es el referente a la aplicabilidad del método por los distintos docentes de derecho de las universidades estudiadas. Al respecto, y teniendo en cuenta el referido concepto del método enunciado, los docentes enseñan por diversas razones, entre las que se destaca: a) por su formación en el extranjero, es decir el hecho de haber realizado cursos de posgrado en universidades de EE. UU., y haber aprendido con esa metodología; b) por su «experiencia vivida» durante su propio trayecto de formación en la carrera de grado, de modo que expresan «enseño de esta manera porque me enseñaron así»; c) por su «socialización profesional», es decir, cuando ingresan a una cátedra universitaria que sigue determinada forma de enseñanza con los alumnos $y$, por ende, adoptan esa metodología que es con la que trabajan año tras año.

Estos son, pues, algunos de los hallazgos obtenidos durante la investigación. Desde los integrantes del proyecto de investigación estamos trabajando en un libro donde abordaremos en profundidad todos estos temas que son de interés para la comunidad universitaria.

\section{REFLEXIONES SOBRE EL MÉTODO DE CASOS Y LA EVALUACIÓN EN CONTEXTO DE PANDEMIA}

Este contexto de pandemia, sobre todo a los docentes del sistema presencial nos impulsó a reflexionar sobre nuestra práctica docente. Antes de la pandemia el aula virtual era un espacio complementario - escasamente utilizado- en la enseñanza, donde el docente subía algún material de lectura, hacía anuncios, subía algún video, artículo, etc. La comunicación se daba «cara a cara». Ahora, el aula virtual es «el» canal de comunicación por excelencia y prácticamente el único con el que contamos. Es un espacio donde hay actividades para hacer, donde se produce el encuentro sincrónico y asincrónico. Sin duda, que este contexto de pandemia nos obliga a replantear la práctica docente y a reflexionar sobre ella, sobre la forma de plantear actividades de aprendizaje, la evaluación, etc. Respecto de la evaluación, tenemos que tener presente que el alumno ya cuenta con ciertos recursos en su domicilio, como libros, Internet, etc. Pero, sobre todo, porque nuestra concepción de evaluación es formativa o de seguimiento. Si adherimos a esta concepción - evaluación como proceso- nuestra forma de entender el proceso de enseñanza y aprendizaje no se reduce a que el alumno reproduzca un conocimiento, sino que además demuestre ciertas habilidades cognitivas y cómo emplea ese conocimiento o contenido.

Esta hibridación entre la presencialidad y la virtualidad, o solamente desde la virtualidad, nos desafía no solo como docentes sino como partes de una misma institución. Ahora tenemos que ser más creativos; no digo que antes no lo fuésemos, sino que tenemos que seleccionar de manera más precisa los recursos pedagógicos que nos permitan llegar a los objetivos propuestos. Cada foro de participación, cada tarea debe ser pensada en pos de preten- 
der que el alumno alcance determinado objetivo, que aprenda cierto concepto y lo aplique o que realice determinado razonamiento. Y aquí aparece la planificación del docente, esta competencia docente que le ayudará a diagramar las distintas actividades. Y esto es importante porque cada actividad está encaminada a que el alumno llegue a determinados objetivos.

$Y$ es en este contexto donde aparece el método de casos y el modelo pedagógico denominado flipped classroom. Este último puede utilizarse tanto en un sistema presencial como blended learning o a distancia. Desde la Cátedra de Introducción al Derecho sistema campus virtual, hemos implementado talleres (webinarios) en los cuales debatimos sobre los distintos casos previamente trabajados por los alumnos de manera individual, utilizando de esta manera ese espacio de encuentro para debatir, compartir y aprender. En este contexto, el rol del alumno es activo, es quien participa, comparte sus puntos de vista, opiniones y el docente es un coordinador; a diferencia del método de enseñanza tradicional, donde solo el docente habla y el alumno tiene una escasa participación. En ese sentido, a la explicación de los contenidos teóricos el alumno accede a través de videos previamente elaborados por el docente. Entonces, los webinarios se convierten en un espacio donde se producen el debate y el aprendizaje crítico sobre la materia. En efecto, en los contextos virtuales se puede lograr en el alumno un aprendizaje significativo. Un docente de derecho constitucional de la Universidad Católica de Salta me comentó sobre este punto - con motivo del proyecto de investigación mencionado-, lo siguiente:

... incluso las plataformas que existen en distancia permiten más que en la presencialidad el trabajo con método de casos porque hay muchas herramientas que se pueden usar en el sistema, como foros (de debate o participativos), herramientas a-sincrónicas que no tenemos en la presencialidad que los sistemas virtuales lo permitan.

Este es un punto de vista interesante, ya que en la plataforma virtual de UCASAL no solo contamos con foros sino también con otros recursos como la wiki colaborativa que permite un trabajo colaborativo; glosarios; collaborate blackboard para las clases en línea (webinarios), etc. Aquí, la planificación del docente es esencial para el logro de buenos resultados de aprendizajes y competencias en los alumnos. Y, sin duda, el acompañamiento institucional a través del personal técnico es de vital importancia para facilitar el proceso de enseñanza y aprendizaje.

\section{CONCLUSIONES}

Pienso que, en estos tiempos de pandemia, el rol del docente se ha ampliado. El docente debe ser un facilitador, coordinador de recursos pedagógicos para que el alumno aprenda. Un docente que trata de hacer pensar, de incentivar, que motiva y enseña a pensar el derecho. Desde este punto de vista, la pandemia puede ser vista como una oportunidad para replantear nuestros modos de enseñanza, y sobre todo una oportunidad para introducir las TIC definitivamente en el aula. No podemos seguir desconfiando de las tecnologías, ya que estas han llegado para quedarse; al contrario, las tecnologías deben ser nuestras aliadas en el proceso educativo. Por último, la pandemia puede ser un buen puntapié inicial para que dejemos de percibir lo virtual como un entorno de no-presencialidad y, en cambio, entendamos a la educación toda como un proceso que se da a partir de la interacción de diver-

Omnia. Derecho y sociedad, núm. 4, 2021: 47-54 
sas formas de copresencia, algunas mediatizadas, otras no-mediatizadas.

\section{REFERENCIAS BIBLIOGRÁFICAS}

Ackerman, M. (2014). Casos y materiales del derecho del trabajo y de la seguridad social. Buenos Aires: Eudeba.

Böhmer, M. (1999). La enseñanza del derecho y el ejercicio de la abogacía. Barcelona: Gedisa.

Carrió, G. (2003). Cómo estudiar y argumentar un caso. Buenos Aires: Lexis Nexis. Abeledo Perrot.

Cueto Rúa, J. (1957). El Common Law. Su estructura normativa. Su enseñanza. Buenos Aires: La Ley.

Fischman, F.; Seda, J. (2011). Formación pedagógica en derecho. Buenos Aires: Miño y Dávila.

Flores, O. (2019). Un método critico de enseñanza y aprendizaje del derecho. Tucumán: Libro digital.

Gordillo, A. (1988). El método en derecho. Madrid: Civitas.

Gullco, H. (2017). Casos de derecho penal especial. Buenos Aires: Ad-Hoc.

Medina, G.; Pitrau, O. (2017). Casos de derecho de familia. Buenos Aires: Abeledo Perrot.

Ovejero Silva, B. (2018). Introducción al Common Law como sistema jurídico. San Miguel de Tucumán.
Palacio, S. (2017). Pedagogía jurídica. Buenos Aires: Estudio.

Piotti, L.; Lupiañez, S. (2002). Análisis institucional y vínculos de convivencia escolar: el desafío de construir el nosotros en las instituciones educativas. Córdoba.

Poggi, M.; Frigerio, G.; Tiramonti, G. (1992). Las instituciones educativas. Cara y ceca. Buenos Aires: Troquel.

Rabbi Baldi Cabanillas, L. R (2019). Lecciones de teoría del derecho. Una visión desde la jurisprudencia constitucional. Buenos Aires: Ábaco.

Rolleri, G.; Pitrau, O. (2017). Casos de derecho sucesorio. Buenos Aires: Astrea.

Sancinetti, M. (2016). Casos de derecho penal. Buenos Aires: Hammurabi.

Suárez, C. (2012). Como plantear, resolver y argumentar un caso. Buenos Aires: García Alonso.

Toller, F. (2005). Orígenes históricos de la educación jurídica con el método de casos. Anuario da Facultade de Dereito Universidade da Coruña, n. ${ }^{\circ}$ 9, 2005. ISSN: 1138039X, ISSN-e 2530-6324, pp. 921-941.

Wassermann, S. (1994). El estudio de casos como método de enseñanza. Argentina: Amorrortu editores.

Ziffer, P. (2011). Casos de derecho procesal penal. Buenos Aires: Hammurabi. 